\title{
Un análisis documental de la investigación en dislexia en la edad adulta*
}

\author{
A Documentary Analysis of Research in Dyslexia in Adulthood
}

Recibido: 12 de marzo de 2015|Aceptado: 04 de marzo de 2016

\author{
MANUEl SORIANO-FERRER ** \\ Universidad de Valencia, España \\ ELISA PIEDRA-MARTÍNEZ *** \\ Universidad de Azuay, Ecuador
}

doi: 10.11144/Javeriana.upsy15-2.adid

Para citar este artículo: Soriano-Ferrer, M., \& Piedra-Martínez, E. (2016). Un análisis documental de la investigación en dislexia en la edad adulta. Universitas Psychologica, 15(2), 193-204.http://dx.doi. org/10.11144/Javeriana.upsy15-2.adid

* Artículo de revisión. Agradecimientos: Este trabajo ha sido realizado al amparo del proyecto de investigación EDU2012-35786 del Ministerio de Ciencia e Innovación (Plan Nacional I+D+I).

** Departamento de Psicología Evolutiva y de la Educación. Facultad de Psicología. Correo electrónico: Manuel.Soriano@uv.es

${ }^{* * *}$ Escuela de Educación Especial, Escuela de Psicología Educativa, Facultad de Filosofía. Correo electrónico: epiedra@uazuay.edu.ec

\section{RES UMEN}

La dislexia en la edad adulta afecta alrededor del $4 \%$ de la población. Por ello, el objetivo de este trabajo es analizar los aspectos metodológicos, así como los temas de investigación, acerca de la dislexia en población adulta. A partir de una búsqueda bibliográfica en la base de datos del Psychological Abstracts: PsycoINFO, se identifican y seleccionan los trabajos sobre los que se centra la revisión. Los resultados indican respecto a la metodología que la mayoría de estudios emplean métodos cuantitativos de investigación (59.4\%), frente al 5.7\% que emplea métodos cualitativos. También se emplean en el 10\% de los trabajos diseños de $\mathrm{N}=1$, y, en menos del 10\%, entrevistas, estudios longitudinales y técnicas neurobiológicas. En cuanto a los temas de investigación, el 40.6\% de los trabajos se centran en analizar procesos cognitivos (conciencia fonológica, memoria, inteligencia, velocidad de procesamiento) y el rendimiento académico (lectura de palabras, lectura de pseudopalabras, ortografía y comprensión de textos), el 25.4\% son estudios neurobiológicos (neurológicos y genéticos), el 12\% se centran en la intervención, el $8 \%$ en identificación y el $5.4 \%$ analizan los problemas asociados a la dislexia en la edad adulta. Los objetivos prioritarios de la investigación parecen ser la caracterización de las manifestaciones de la dislexia en la edad adulta, seguidos de los estudios que se dirigen al esclarecimiento de las bases neurobiológicas. También se amplía la investigación hacia temas de orden práctico.

Palabras clave

dislexia; adultez; análisis documental; revisión

\section{A B S T R ACT}

Dyslexia in adulthood affects around $4 \%$ of the population. Thus, this study offers an overview of the methodological aspects and the areas of research in dyslexia in adulthood. From a bibliographical search conducted in the Psychological Abstracts database: PsycINFO, we identified and selected the studies the review was to focus on. Results show in relation to methodological aspects, quantitative research methods are preferred in $59.4 \%$ of cases, whereas $5.7 \%$ opted for qualitative methods. Also, in $10 \%$ of the studies, used $\mathrm{N}=1$ designs and in an under $10 \%$ studies used surveys, longitudinal/ follow-up studies or neurobiological procedures. In relation to the topics of research, $40.6 \%$ analyzed cognitive processes (phonemic awareness, memory, IQ, naming speed) and/or academic achievement (word reading, pseudoword reading, spelling, and reading comprehension), the $25.4 \%$ we- 
re neurobiological studies (neurological and genetics), the $12 \%$ were studies focusing on intervention, the $8 \%$ focused on identification/ diagnostic, and 5.4\% analyzed associated problems to dyslexia in adulthood. The primary objectives of the research appear to be the characterisation of dyslexia and studies about neurobiological basis of dyslexia in adulthood. Research is also extended towards other, more practical matters.

Keywords

dyslexia; adulthood; documentary analysis; review

\section{Introducción}

La dislexia evolutiva constituye un síndrome heterogéneo de origen neurológico que persiste a lo largo del ciclo vital. Se caracteriza por un fracaso inesperado en el reconocimiento de las palabras que se manifiesta en la incapacidad para desarrollar una lectura fluida y sin esfuerzo (Serrano \& Defior, 2008; Fletcher, 2009; Peterson \& Pennington, 2012; Snowling \& Hulme, 2012; Shaywitz, Morris, \& Shaywitz, 2008; Soriano \& Miranda, 2010). Por supuesto, una de las más consistentes y centrales características es su concepción como una dificultad en la lectura de carácter inesperado, que se refiere a la presencia de una dificultad en la lectura en un niño (o adulto) que parece tener todo lo necesario (inteligencia, motivación, instrucción adecuada) para ser un buen lector y, sin embargo, continúa presentando dificultades (Shaywitz et al, 2008). Como destaca el DSM-5 la dislexia "no se explica mejor por discapacidades intelectuales, trastornos visuales o auditivos no corregidos, otros trastornos mentales o neurológicos, adversidad psicosocial, falta de dominio en el lenguaje de instrucción académica o directrices educativas inadecuadas" (APA, 2014, p. 67). Junto a las dificultades en el reconocimiento exacto o fluido de palabras, se señalan dificultades en la ortografía, así como en la comprensión lectora y razonamiento matemático (APA, 2014).

La prevalencia de la dislexia se ha estimado en un $5 \%-15 \%$ de los niños en edad escolar dependiendo de la lengua y cultura (APA, 2014). En concreto, en español, dependiendo del criterio empleado, se ha estimado una prevalencia que oscila entre el 3.2\% y el 5.9\% en la enseñanza primaria (Jiménez, Gúzman, Rodríguez, \& Artiles, 2009) y entre el 3.2 y el $5.6 \%$ en la enseñanza secundaria (González et al., 2010). Aunque no existen estudios de prevalencia en la edad adulta, se considera que es de alrededor del 4\% (APA, 2014).

Por otro lado, diversas investigaciones longitudinales han mostrado que las dificultades lectoras constituyen un trastorno crónico, con fuerte persistencia de las dificultades lectoras en la adolescencia y en la edad adulta (Bruck, 1990, 1992; Lesaux, 2001; Shaywitzet al., 1999; Snowling, Muter, \& Carroll, 2007; Swanson \& Hsieh, 2009; Undheim, 2009). Resultan clarificadoras las conclusiones del metaanálisis de Swanson \& Hsieh (2009) sobre las manifestaciones de la dislexia en la edad adulta en lengua inglesa. Los adultos con dislexia presentan claras dificultades en las tareas de procesamiento fonológico, velocidad de procesamiento y de memoria verbal, así como un menor desempeño en tareas de lectura y escritura. Además, como destaca Swanson (2012), las deficiencias de los adultos con dislexia son más severas en aquellos con mayor CI. Aunque escasos, los estudios realizados en adultos con dislexia en lenguas más transparentes que el inglés evidencian mayores dificultades a nivel de ortografía que de lectura (Nergård-Nilssen \& Hulme, 2014; Re, Tressoldi, Cornoldi, \& Lucangeli, 2011; Tops, Callens, Lammertyn, Van Hees \& Brysbaert, 2012).

De hecho, no resulta extraño que los adultos con dislexia dediquen menos tiempo a las actividades de lectura, adquiriendo menos vocabulario y comprometiendo seriamente la comprensión lectora. Especiales dificultades presentan en la comprensión de textos complejos y en la realización de inferencias todavía los adultos con dificultades lectoras (Simmons \& Singleton, 2000). Por ello, los jóvenes y adultos con dificultades lectoras evidencian una carencia de conocimientos con respecto a las personas sin dificultades, lo cual puede mermar seriamente su desempeño en tareas de lectura.

A partir de estas consideraciones previas, el presente estudio trata de ofrecer un punto de vista general sobre la evolución y estado actual de la investigación en dislexia en la edad adulta, basado 
en una selectiva revisión de la literatura publicada sobre este tema en los últimos años. Mediante un análisis cuantitativo sistemático de las publicaciones tratamos de: (a) sintetizar, integrar y discutir, desde un punto de vista diacrónico, los aspectos metodológicos empleados en la investigación sobre la dislexia en adultos, así como (b) los tópicos sobre los que se ha centrado dicha investigación durante los últimos seis años.

\section{Metodología}

El proceso de identificación y selección de los estudios incluidos en nuestra revisión se llevó a cabo a partir de una búsqueda bibliográfica en la base de datos computarizada del Psychological Abstracts PsycINFO. Con el propósito de orientar la búsqueda informática en la dirección deseada, empleamos los diferentes términos que incluye el Tesauro utilizados para clasificar los documentos (dyslexia, reading disabilities, reading learning disabilities). De este modo se pretendía localizar estudios cuyo título, resumen (abstract) o índice terminológico incluyera los conceptos seleccionados.

En concreto, se realizaron dos tipos de búsqueda, una dirigida a la realización de una aproximación metodológica en la investigación en dificultades lectoras en población adulta y la otra dirigida a mostrar objetivamente aquellos temas sobre los que se centra dicha investigación. En relación con nuestro primer objetivo, se realizó una búsqueda dirigida a seleccionar todos los artículos publicados sobre dificultades de aprendizaje lector en adultos, considerando artículos de revistas científicas, textos, tesinas-tesis doctorales, revisados por pares y definiendo el rango de edad en más de 18 años. Con el fin de mostrar la evolución diacrónica de las metodologías de estudio empleadas en la investigación sobre el tema se realizaron tres búsquedas correspondientes a tres grandes periodos temporales: antes de 1988, de 1989 a 2001 y de 2002 a 2014.

Por otro lado, en relación con nuestro segundo objetivo, destinado a exponer la temática que aborda la investigación de la dislexia en adultos en los últimos años, se realizó un análisis documental de contenido (Pinto, 2002). Para ello, seleccionamos las bases de datos correspondientes a los últimos seis años (2009-2014) y, tras introducir las palabras clave, se localizaron los trabajos. Se seleccionaron exclusivamente los artículos, eliminado de nuestra revisión los libros, tesis, tesinas, capítulos de libro $\mathrm{o}$ actas de congreso o reuniones científicas. A continuación, se leía el resumen y se consultaban los descriptores que se emplean en dicha base de datos como mecanismos de indización. Con este procedimiento se seleccionaron los trabajos que formarían parte de nuestra revisión y se eliminaron aquellos que no se centraban en las dificultades lectoras en adultos, o aquellos en los la población adulta con dificultades lectoras no era el tema central del artículo. Acto seguido se procedió a la categorización jerárquica de cada uno de los trabajos por temas o áreas y a su agrupación en virtud de la categoría de pertinencia y, posteriormente, al recuento de frecuencias de cada categoría. En aquellos trabajos que suscitaban dudas, que fueron bastantes, se optó por un sistema de jueces (dos profesores universitarios) como criterio decisivo.

\section{Resultados}

\section{Aspectos metodológicos en la investigación en dislexia en población adulta}

A partir de las fuentes mencionadas, se calculó el porcentaje de trabajos publicados para cada uno de los tipos de estudio en cada uno de los periodos (antes de 1988, 1989 a 2001, 2002 a 2014) en los que se centró nuestra revisión. El número de estudios que se realizan en la investigación en dislexia en población adulta ha variado sustancialmente en los últimos años. En concreto, del total de 3142 trabajos localizados, el 64.3\% $(n=2019)$ de los trabajos se han publicado en el periodo 2002-2014, mientras que el $26 \%(n=817)$ se publicó en el periodo de 1989-2001 y sólo el 9.7\% (n=306) se publicó con anterioridad a 1988. De forma que es en los últimos trece años donde se ha duplicado el número de trabajos sobre dislexia en adultos.

Con el fin de mostrar la evolución diacrónica de las metodologías empleadas en la investigación sobre dislexia en población adulta, se hizo un cálculo 
para cada uno de los tres periodos de tiempo en los que se ha centrado nuestra revisión. Es necesario precisar que los estudios pueden estar clasificados en dos tipos de metodología al mismo tiempo. Así, por ejemplo, algunos estudios aparecen en la categoría "estudio longitudinal" junto con "estudio cuantitativo". Los resultados aparecen en el gráfico 1 agrupados en los tres periodos analizados. Como puede observarse en este gráfico, del total de estudios localizados ( $n=3142), 1865$ estudios han empleado metodología cuantitativa (59.4\%). De éstos, 984 (52.7\%) se han publicado en los últimos trece años (2002-2014), 634 (34\%) en el periodo anterior (1989-2001) y sólo 247 (13.2\%) han sido publicados antes de 1988.

Por el contrario, la metodología cualitativa ha sido empleada en sólo 182 de los estudios realizados en adultos (5.7\%), aunque como puede observarse, el 98.5\% ( $n=179)$ de estos estudios ha sido realizado en los últimos trece años. En la misma línea se muestran los estudios que han empleado entrevistas ( $\mathrm{n}=237 ; 7.5 \%)$, de las que también el $98.4 \%(\mathrm{n}=233)$ se han publicado en el último periodo analizado (2002-2014). Posiblemente esta escasa utilización de metodologías cualitativas frente a las cuantitativas se deba a que la investigación se ha centrado fundamentalmente en el estudio de las diferencias individuales ocasionadas por alteraciones neurológicas o cognitivas de los adultos con dislexia. $\mathrm{Ob}$ viamente, la metodología cualitativa, más enfocada al estudio de la influencia del contexto social, las autopercepciones o fortalezas y debilidades de los adultos con dislexia, se muestra inadecuada en esta línea de trabajo.

En relación a los estudios de $\mathrm{N}=1$, en el que agrupamos tanto los estudios de caso clínico como



Gráfico 1. Evolución de la metodología de estudio de la dislexia en adultos.

Fuente: elaboración propia. 
los estudios de caso no clínicos, se han publicado un total de 320 (10.2\%), de los que el 86.6\% se ha publicado en los últimos 26 años. En concreto, el $50.6 \%(n=162)$ se ha publicado entre 2002 y 2014 , el $36 \%(n=115)$ se ha publicado entre 1989 y 2001 , mientras que el $13.4 \%(n=43)$ se ha publicado con anterioridad a 1988.

Otro aspecto destacable es la preocupación de los investigadores por lograr una buena comprensión de la dislexia en la edad adulta y poder especificar los mecanismos causales subyacentes a ésta, como se desprende del hecho de que cada vez van teniendo más presencia los estudios longitudinales o de seguimiento ( $\mathrm{n}=231 ; 7.3 \%)$. De hecho, el $79.7 \%(n=184)$ se ha publicado en el último periodo (2002-2014), mientras que $16.8 \%$ se publicó en el periodo anterior (1989-2001) y sólo el 3.5\% $(n=8)$ se publicó antes de 1988. Además, el 100\% $(n=31)$ de los estudios de tipo prospectivo, al igual que el 95.2\% $(n=20)$ de los estudios de tipo retrospectivo, se ha realizado en el último periodo (2002-2014), no existiendo ningún estudio de este tipo con anterioridad a 1988.

También aparecen con fuerza los estudios neurobiológicos (estudios genéticos y neurológicos). Así, 192 (6.1\%) estudios se han dirigido al esclare- cimiento de la etiología de la dislexia en adultos. En concreto, el 95.8\% $(n=184)$ de los mismos se ha publicado en el último periodo analizado (2002-2014).

Otro tema que continúa interesando a los investigadores es la revisión de la literatura $(n=21)$, que muestra un ligero aumento en el último periodo. Este tipo de estudios intenta realizar una síntesis cualitativa y cuantitativa de los resultados de los diferentes estudios independientes y valorar el conjunto de resultados de todos los estudios que aparentemente han investigado sobre la misma cuestión. Así, las revisiones de la literatura e investigación pasan del 42.8\% (n=9) entre 1989 y 2001 al 57.1\% $(n=12)$ entre 2002 y 2014. Una tendencia similar muestran las revisiones meta-analíticas $(\mathrm{n}=12)$, que pasan del $41.6 \%(n=5)$ entre 1989 y 2001 al $58.3 \%$ $(n=7)$ entre 2002 y 2014.

En la misma línea, se manifiesta la preocupación entre los especialistas en dislexia por determinar con exactitud la eficacia relativa y diferencial de las intervenciones o acomodaciones educativas $(n=28)$, de las que el $82.3 \%(n=23)$ se ha publicado en los últimos trece años (2002-2014), lo que pone de manifiesto el interés y necesidades de apoyo a los disléxicos en las diferentes etapas de la vida.

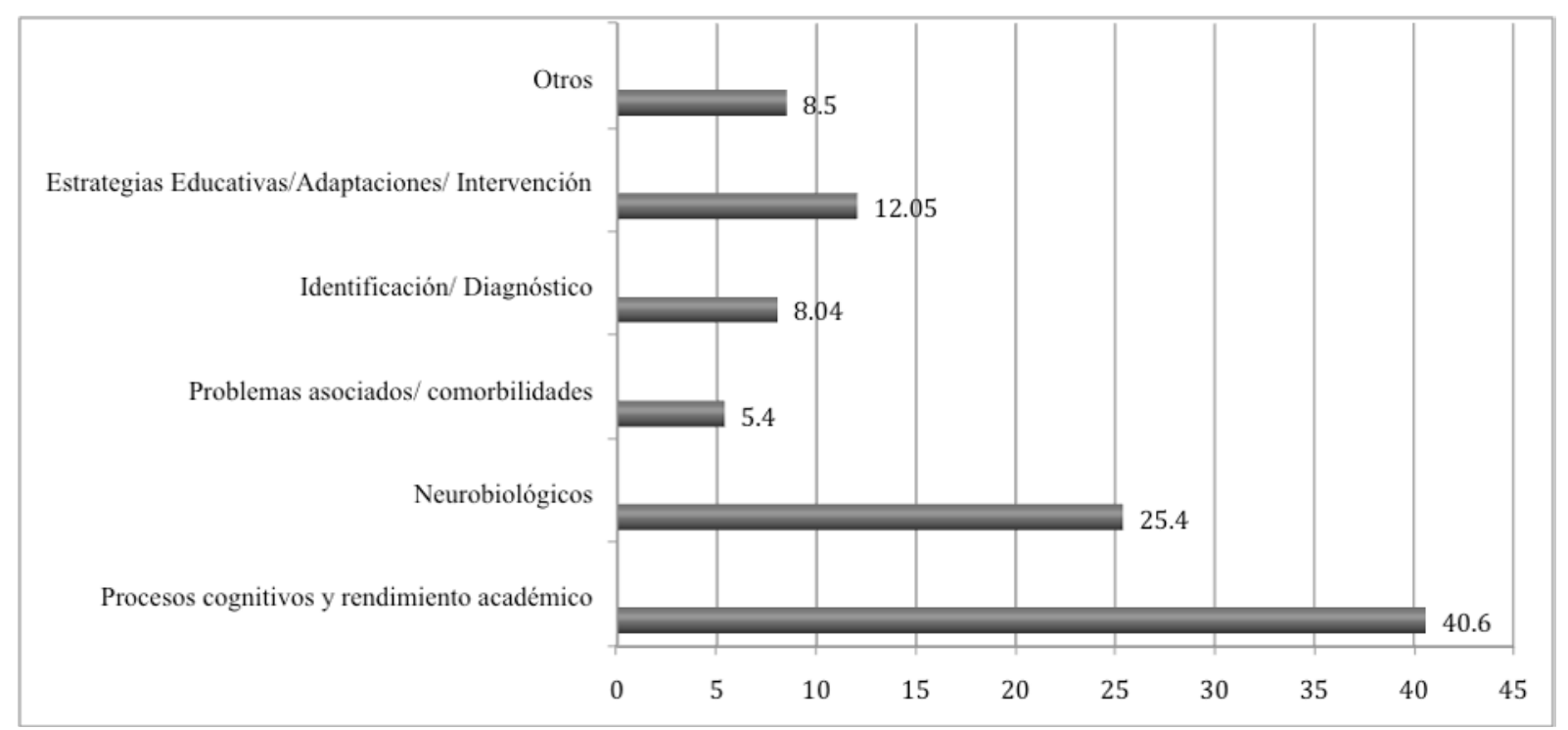

Gráfico 2. Análisis de contenido de la investigación en dislexia entre 2009 y 2014

Fuente: elaboración propia. 
Análisis de contenido de investigación en dislexia en población adulta

Con el fin de precisar la tendencia actual de la investigación de la dislexia en la edad adulta, se ha realizado una revisión de las publicaciones durante los últimos seis años. Así, siguiendo el procedimiento expuesto anteriormente, se localizaron 224 artículos que utilizan en sus estudios muestras de adultos disléxicos. A continuación, a partir de resumen o del artículo completo, se procedió a la categorización jerárquica de cada uno de los trabajos por temas o áreas, a su agrupación en virtud de la categoría de pertinencia y, posteriormente, al recuento de frecuencias de cada categoría. Así, se clasificaron los artículos en seis categorías (véase gráfico 2) que integran la amplitud de temas tratados dentro de este campo.

Además de aquellas categorías que engloban un mayor número de trabajos, se realizó a su vez una clasificación jerárquica por tópicos incluidos en dichos trabajos (véase tabla 1). Así, el 40.6\% de los trabajos ( $\mathrm{n}=91$ ) se centró en el análisis de los procesos cognitivos y en el rendimiento académico. Dentro de esta amplia categoría, 21 trabajos analizaron exclusivamente los procesos cognitivos de los disléxicos en la edad adulta, once se centraron en el análisis del rendimiento académico y la mayoría de ellos $(\mathrm{n}=59)$ analizaron ambos aspectos al mismo tiempo. En relación a los trabajos sobre los procesos cognitivos de los adultos con dislexia ( $\mathrm{n}=75 ; 82.4 \%)$, los procesos cognitivos más analizados en los diferentes trabajos han sido: la conciencia fonológica $(\mathrm{n}=45 ; 60 \%)$; la memoria $(\mathrm{n}=42 ; 56 \%)$; la velocidad de procesamiento y la inteligencia ( $\mathrm{n}=30 ; 40 \%)$; la percepción y atención visuales y los movimientos oculares $(n=28 ; 37.3 \%)$; el vocabulario $(n=19$; 25.3\%); la percepción y atención auditiva, percepción de habla y ritmo ( $\mathrm{n}=15 ; 20 \%$ ), y, en menor medida, las habilidades motrices $(\mathrm{n}=8 ; 10.7 \%)$, la fluidez verbal $(\mathrm{n}=6 ; 8 \%)$ y el funcionamiento ejecutivo $(n=4 ; 5.3 \%)$. Por su parte, de los trabajos que analizan el rendimiento académico de los adultos con dislexia $(\mathrm{n}=64 ; 70.3 \%)$, la mayoría analizan el rendimiento en lectoescritura $(\mathrm{n}=61 ; 95.3 \%)$ y un pequeño porcentaje de ellos analiza el rendimiento en matemáticas ( $\mathrm{n}=8 ; 12.5 \%$ ). De los trabajos que han analizado la lectoescritura, el $77.1 \%(n=47)$ ha analizado la lectura de palabras aisladas, el 60.6\% ha analizado la lectura de pseudopalabras $(n=37)$ y la escritura de palabras-ortografía ( $n=37$ ), el $55.7 \%$ ha analizado la velocidad o exactitud de los disléxicos adultos en la lectura de un texto $(n=34)$. En menor medida, el 24.6\% de los trabajos han analizado los procesos de comprensión de textos ( $\mathrm{n}=15)$, el $8.2 \%$ la escritura de pseudopalabras $(n=5)$ y el $5 \%$ los procesos de composición de textos $(\mathrm{n}=3)$.

La segunda de las líneas de investigación más importantes en los últimos años se ha centrado en el análisis de los sustratos neurobiológicos de la dislexia en población adulta, que representa el $25.4 \%(n=57)$. Dentro de los estudios que han empleado técnicas de neuroimagen, el $35.1 \%(n=20)$ ha empleado técnicas neurofisiológicas, el 26.3\% ha empleado técnicas neurofuncionales $(n=15)$ y el 24.5\% ha empleado técnicas neuroestructurales $(\mathrm{n}=14)$. Por su parte, el $28.1 \%$ de los estudios se ha dirigido al análisis genético $(\mathrm{n}=16)$, siendo el $62.5 \%$ estudios de genética molecular $(n=10)$ y el $37.5 \%$ de genética cuantitativa-heredabilidad $(\mathrm{n}=6)$.

En orden decreciente, el $12.05 \%$ de los trabajos ( $\mathrm{n}=27$ ) se ha centrado en el análisis de diferentes estrategias educativas-adaptaciones y procedimientos de intervención, el $8.04 \%$ se ha centrado en procedimientos de identificación o diagnóstico $(\mathrm{n}=18)$, el $5.4 \%$ se ha centrado en problemas asociadoscomorbilidades de los adultos con dislexia $(\mathrm{n}=12)$. Por último, en la categoría otros $(\mathrm{n}=19 ; 8.5 \%)$ hemos agrupado trabajos de temáticas dispares como relación con drogas, análisis de políticas, o uso de Facebook, entre otros.

\section{Conclusiones}

El análisis de la literatura en dificultades lectoras en población adulta señala un notorio incremento de la investigación, especialmente desde el año 2002, sobre todo con métodos cuantitativos, que son los preferidos por los investigadores.

Un objetivo prioritario de la investigación en los últimos años se centra en averiguar cuáles son las dificultades persistentes en los disléxicos adultos 
TABLA 1

Análisis de contenido de la investigación en dislexia en adultos entre 2009 y 2014

\begin{tabular}{|c|c|c|}
\hline & $\mathrm{N}=224$ & $\%$ \\
\hline a.- Procesos cognitivos y rendimiento académicoa & 91 & 40.6 \\
\hline Procesos cognitivos & 75 & 82.4 \\
\hline Inteligencia & 30 & 40 \\
\hline Conciencia fonológica & 45 & 60 \\
\hline Velocidad de procesamiento & 30 & 40 \\
\hline Vocabulario & 19 & 25.3 \\
\hline Memoria a corto plazo y de trabajo & 42 & 56 \\
\hline Percepción y atención visuales y movimientos oculares & 28 & 37.3 \\
\hline Percepción y atención auditivas, percepción de habla y ritmo & 15 & 20 \\
\hline Habilidades motrices & 8 & 10.7 \\
\hline Fluidez verbal & 6 & 8 \\
\hline Funcionamiento ejecutivo & 4 & 5.3 \\
\hline Rendimiento académico & 64 & 70.3 \\
\hline Lectoescritura & 61 & 95.3 \\
\hline Reconocimiento de palabras (aisladas) & 47 & 77.1 \\
\hline Lectura en texto (velocidad y exactitud) & 34 & 55.7 \\
\hline Lectura de pseudopalabras & 37 & 60.6 \\
\hline Comprensión de textos & 15 & 24.6 \\
\hline Escritura de palabras /ortografía & 37 & 60.6 \\
\hline Escritura de Pseudopalabras & 5 & 8.2 \\
\hline Composición de textos & 3 & 5 \\
\hline Matemáticas & 8 & 12.5 \\
\hline b.-Neurobiológicosa & 57 & 25.4 \\
\hline Funcionales & 15 & 26.3 \\
\hline Estructurales & 14 & 24.5 \\
\hline Fisiológicos & 20 & 35.1 \\
\hline Genéticos & 16 & 28.1 \\
\hline Molecular & 10 & 62.5 \\
\hline Heredabilidad & 6 & 37.5 \\
\hline c.- Estrategias Educativas/Intervención/Adaptaciones & 27 & 12.05 \\
\hline d.- Identificación/Diagnóstico & 18 & 8.04 \\
\hline e.- Problemas asociados/comorbilidades & 12 & 5.4 \\
\hline f.- Otros & 19 & 8.5 \\
\hline
\end{tabular}

a. Los porcentajes superan el 100\% debido a que los trabajos analizan varios aspectos al mismo tiempo.

Fuente: elaboración propia.

a nivel de procesos cognitivos, así como las manifestaciones académicas a nivel de los aprendizajes instrumentales, es decir, la prioridad parece ser el averiguar con exactitud cuáles son las manifestaciones cognitivas y de rendimiento lectoescritor de las dificultades lectoras en la edad adulta. Especial importancia cobran los estudios translingüísticos dirigidos a la caracterización de la dislexia en adul- tos en las diferentes lenguas, ya que las variaciones que se presentan dentro de una ortografía están relacionadas con las características de las dificultades lectoras. Así, frente a las lenguas opacas como el inglés, los adultos con dislexia en las lenguas transparentes como el italiano, el noruego, el finlandés parecen caracterizarse más por las dificultades con la fluidez lectora y las dificultades con la ortografía 
(Nergård-Nilssen \& Hulme, 2014; Re et al., 2011; Tops et al., 2012).

Por otro lado, dado que el peso de las variables predictoras del éxito en el aprendizaje de la lectura es diferente en las lenguas de diferente regularidad, es de suma importancia conocer si dichos predictores se mantienen estables a largo plazo, es decir, en la edad adulta, en las diferentes lenguas. Como sugieren Nergård-Nilssen \& Hulme (2014) existen buenas razones para asumir que las deficiencias cognitivas observadas en los adultos con dislexia han persistido desde la infancia.

Otra de las líneas de investigación importantes de los últimos años son los estudios neurobiológicos dirigidos a clarificar la etiología de la dislexia. Así, las técnicas de neuroimagen están permitiendo un mayor conocimiento acerca de las diferencias neurológicas en los adultos con dislexia, como son una menor activación de áreas relacionadas con las redes frontales, áreas temporoparietales y, especialmente, con la zona visual de la palabra (disfunción occipitotemporal) en el hemisferio izquierdo, así como diferencias volumétricas en materia blanca y gris en esas zonas. También los estudios de genética cuantitativa y de genética molecular destacan la alta heredabilidad de diferentes fenotipos disléxicos, así como la implicación de diferentes genes en la dislexia en la edad adulta (véase revisión en SorianoFerrer \& Piedra-Martínez, 2014).

Otro grupo de trabajos parece estar centrado en las estrategias y acomodaciones educativas o procedimientos de intervención, ya sea a los procesos académicos, sociales o emocionales en los adultos disléxicos, especialmente estudiantes universitarios. Todos estos trabajos subrayan la importancia de conocer las dificultades actuales de los adultos para establecer estrategias de apoyo y prevención (Callens, Tops, \& Brysbaert, 2012). Algunos trabajos proponen acomodaciones educativas o laborales (p.e., uso de sintetizadores de voz en la lectura, grabaciones, compañero lector, aumento de tiempo, etc.) para adolescentes y adultos, mientras que otros analizan la eficacia de procedimientos concretos de intervención en lectura (Lingo, 2014; Lovett, Lacarenza, Steinbach, \& De Palma, 2014) e incluso la eficacia de un software de autocorrec- ción sobre la comprensión (Hiscox, Leonavičiūtè, \& Humby, 2014).

Otra novedosa tendencia consiste en la aplicación de las técnicas de neuroimagen en estudios de intervención. Como manifiesta la revisión metaanalítica de Barquero, Davis, \& Cutting (2014) los hallazgos de estos estudios encuentran diferencias funcionales en la activación de diferentes áreas involucradas en la lectura, aunque destacan la necesidad de tomar estos datos con cautela debido al escaso número de trabajos y las discrepancias entre los estudios. No obstante, estos cambios en la activación cerebral representan la firma biológica que respalda contundentemente la validación de las intervenciones.

Otro de los aspectos que suscita mucho interés entre los investigadores está relacionado con la identificación y diagnóstico de los adultos con dislexia. Algunos trabajos se han centrado en las propiedades psicométricas de instrumentos de evaluación en población adulta (Gregg et al., 2008; Nanda, Greenberg, \& Morris, 2014; Díaz, Jiménez, Mejía, \& Fabregat, 2014). Pero sin duda, la mayoría de trabajos se ha centrado en el desarrollo o adaptación de autoinformes acerca de la historia de las dificultades lectoras de los adultos con dislexia. Por ejemplo, Lefly \& Pennington (2000), en el estudio de validación de un cuestionario para adultos (Adults Reading History Questionnaire ARHQ) concluyeron que la autovaloración de los participantes coincidía en un alto porcentaje con las medidas obtenidas mediante pruebas de evaluación. Por su parte, Wolff \& Lundberg (2003) confirman que el autoinforme es el elemento que mejor discrimina entre los participantes adultos con y sin problemas de lectura. Por su parte, Deacon, Cook, $\&$ Parrila (2012) confirmaron que los universitarios disléxicos diagnosticados en la infancia, así como los universitarios identificados mediante autoinforme, puntuaron más bajo que los controles en pruebas precisión y velocidad de lectura. En Islandia, Bjornsdottir et al. (2014) empleando el ARHQ lograron discriminar correctamente el $84.5 \%$ de los disléxicos adultos. Igualmente, en castellano, Giménez, Luque, López-Zamora, \& Fernández (2015) desarrollan un autoinforme de trastornos lectores 
para adultos (ATLAS) que logra discriminar entre normolectores y estudiantes con dificultades. Sin embargo, como destaca el DSM-5 "en individuos de 17 y más años, la historia documentada de las dificultades del aprendizaje se puede sustituir por la evaluación estandarizada" (APA, 2014, p. 67).

Aunque el análisis de los problemas asociados a las dificultades de aprendizaje representa el tópico más investigado del total de la investigación realizada entre 1998 y 2003 (Soriano -Ferrer, 2005), en el caso de los adultos con dislexia representa un tópico minoritario, pero en auge en estos momentos. Así, algunos trabajos se centran en las experiencias personales y necesidades socioemocionales y educativas de los disléxicos adultos en su desempeño personal, familiar, social y laboral (Pino \& Mortari, 2014) y en la incidencia de la etiqueta de dislexia a nivel personal (Macdonald, 2010). Además, las dificultades lectoras afectan negativamente al plano afectivo-motivacional. Por ejemplo, suelen experimentar niveles más altos de ansiedad y sintomatología depresiva (Marwood \& Hewitt, 2013; Nelson \& Gregg, 2012). De hecho, el meta-análisis realizado por Klassen, Tze, \& Hannok (2013) constata cómo los problemas internalizantes son una constante entre los disléxicos en la edad adulta, así como la baja autoestima (Eggleston, Hanger, Frampton, \& Watkins, 2012), la escasa motivación intrínseca (Łockiewicz, Bogdanowicz, \& Bogdanowicz, 2014) y las bajas expectativas, tanto parentales como propias (Rimkute, Torppa, Eklund, Nurmi, \& Lyytinen, 2014). Por su parte, el trabajo de Jordan, McGladdery, \& Dyer (2014) muestra en universitarios con dislexia que experimentan niveles muy altos de ansiedad hacia las matemáticas, siendo los niveles de ansiedad y depresión más altos entre las mujeres con dislexia (Nelson \& Gregg, 2012).

\section{Referencias}

American Psychiatric Association (APA) (2014). Manual diagnóstico y estadístico de los trastornos mentales. DSM-5. [Diagnostic and Statistical Manual of Mental Disorders, Five Edition] Madrid: Editorial Médica Panamericana.
Barquero, L. A., Davis, N., \& Cutting, L. E. (2014). Neuroimaging of reading intervention: A Systematic Review and Activation Likelihood Estimate Meta-Analysis. PLoS ONE, 9(1), e83668.

Bjornsdottir, G., Halldorsson, J. G., Steinberg, S., Hansdottir, I., Kristjansson, K., Stefansson, H., \& Stefansson, K. (2014). The adult reading history questionnaire (ARHQ) in icelandic: Psychometric properties and factor structure. Journal of Learning Disabilities, 47(6), 532-542.

Bruck, M. (1990). Word-recognition skills of adults with childhood diagnoses of dyslexia. Developmental Psychology, 26(3), 439-454.

Bruck, M. (1992). Persistence of Dyslexic' phonological awareness deficits. Developmental Psychology, 28, 874-886.

Callens, M., Tops, W., \& Brysbaert, M. (2012). Cognitive profile of students who enter higher education with an indication of dyslexia. PLoS ONE, 7(6), e38081.

Deacon, H., Cook, K., \& Parrila, R. (2012). Identifying high-functioning dyslexics: is self-report of early reading problems enough?. Annals of Dyslexia, 62, 120-134.

Díaz, A., Jiménez, J. E., Mejía, C., \& Fabregat, R. (2014). Propiedades psicométricas de la batería de evaluación de procesos cognitivos y de lectura en adultos con dislexia (BEDA). International Journal of developmental and Educational Psychology, 1(1), 565-570.

Eggleston, M., Hanger, N., Frampton, C., \& Watkins. (2012) Coordination difficulties and self-steem: A review and findings from a New Zealand survey. Australian Occupational Therapy Journal, 59, 456-462.

Fletcher, J. M. (2009). Dyslexia: The evolution of a scientific concept. Journal of International Neuropsychological Society, 15, 501-508.

Giménez, A., Luque, J. L., López-Zamora, M., \& Fernández, M. (2015). Autoinforme de Trastornos Lectores para Adultos (ATLAS). Anales de Psicología, 31(1), 109-119.

González, D., Jiménez, J. E., García, E., Díaz, A., Rodríguez, C., Crespo, P., \& Artiles, C. (2010). Prevalencia de las dificultades específicas de aprendizaje en la Enseñanza Secundaria Obligatoria. European Journal of Education and Psychology, 3 (2), 317-327. 
Gregg, N., Bandalos, D. L., Coleman, C., Davis, J. M., Robinson, K., \& Blake, J. (2008). The validity of a battery of phonemic and orthographic awareness tasks for adults with and without dyslexia and attention Deficit/Hyperactivity disorder. Remedial and Special Education, 29(3), 175-190.

Hiscox, L., Leonavičiūtė, E., \& Humby, T. (2014). The effects of automatic spelling correction software on understanding and comprehension in compensated dyslexia: Improved recall following dictation. Dyslexia, 20, (3), 208-224.

Jiménez, J. E., Guzmán, R., Rodríguez, C., \& Artiles, C., (2009) Prevalencia de las dificultades específicas de aprendizaje: La dislexia en español. Anales de psicología, 25, 63-84

Jordan, J., McGladdery, G., \& Dyer, K. (2014). Dyslexia in higher education: Implications for maths anxiety, statistics anxiety and psychological well-being. Dyslexia, 20, (3), 225-240.

Klassen, R. M., Tze, V. M., \& Hannok, W. (2013). Internalizing Problems of Adults With Learning Disabilities A Meta-Analysis. Journal of learning disabilities, 46, (4), 317-327.

Lefly D. L, \& Pennington, B. F. (2000). Reliability and Validity of the Adult Read-ing History Questionnaire. Journal of Learning Disability, 33, 286-296.

Lesaux, N. K. (2001). Persistence of phonological processing deficits in college dyslexics with age-appropriate reading skills. Journal of Learning disabilities, $34,394-400$

Lingo, A. S. (2014). Tutoring Middle School students with disabilities by high school students: Effects on oral reading fluency. Education and Treatment of Children, 37(1), 53-75.

Łockiewicz, M., Bogdanowicz, K. M., \& Bogdanowicz, M. (2014). Psychological resources of adults with developmental dyslexia. Journal of Learning Disabilities, 47(6), 543-555.

Lovett, M. W., Lacarenza, L., Steinbach, K. A., \& De Palma, M. (2014). Development and Evaluation of a Research-based intervention program for children and adolescents with reading disabilities. Perspectives on Language and Literacy, 40(3), 21-31.

Macdonald, S. J. (2010). Towards a social reality of dyslexia. British Journal of Learning Disabilities, 38(4), 271-279.
Marwood, H., \& Hewitt, O. (2013). Evaluating an anxiety group for people with learning disabilities using a mixed methodology. British Journal of Learning Disabilities, 41(2), 150-158.

Nanda, A. O., Greenberg, D., \& Morris, R. D. (2014). Reliability and validity of the CTOPP elision and blending words subtests for struggling adult readers. Reading and Writing, 27, 1603-1618.

Nelson, J. M., \& Gregg, N. (2012). Depression and anxiety among transitioning adolescents and college students with ADHD, Dyslexia, or comorbid ADHD/Dyslexia. Journal of Attentional Disorders, 16(3), 244-254.

Nergård-Nilssen, T., \& Hulme, C. (2014). Developmental Dyslexia in Adults: Behavioural Manifestations and Cognitive Correlates. Dyslexia, 20, 191-207

Peterson, R.L. \& Pennington, B.F. (2012). Developmental dyslexia. The Lancet, 379, 1997-2007.

Pino, M., \& Mortari, L. (2014). The inclusion of students with dyslexia in higher education: A systematic review using narrative synthesis. Dyslexia, 20, 346-369.

Pinto, M., (2002). Análisis Documental de contenido. In Tortosa FM, Civera C. (Eds.), Nuevas tecnologías de la información y documentación en psicología (pp. 81-101). Madrid: Ariel.

Re, A. M., Tressoldi, P. E., Cornoldi, C., \& Lucangeli, D. (2011). Which tasks best discriminate between dyslexic university students and controls in a transparent language? Dyslexia, 17(3), 227-241.

Rimkute, L., Torppa, M., Eklund, K., Nurmi, J., \& Lyytinen, H. (2014). The impact of adolescents' dyslexia on parents' and their own educational expectations. Reading and Writing, 27(7), 1231-1253.

Serrano, F., \& Defior, S. (2008). Dyslexia speed problems in a transparent orthography. Annals of Dyslexia, 58, 81-95.

Shaywitz, S., Fletcher, J., Holahan, J., Shneider, A., Marchione, K., Stuebing, K. K., Francis, D. J., Pugh, K. R., \& Shaywitz, B. A. (1999). Persistence of dyslexia: the Connecticut Longitudinal Study at adolescence. Pediatrics, 104, 1351-59

Shaywitz, S. E., Morris, R., \& Shaywitz, B. A. (2008). The education of dyslexic children from childhood to young adulthood. Annual Review of Psychology, $59,451-475$. 
Simmons, F., y Singleton, C. (2000). The reading comprehension abilities of dyslexic students in higher education. Dyslexia, 6(3), 178-192.

Snowling, M. J., \& Hulme, Ch. (2012). Children's reading impairments: From theory to practice. Japanese Psychological Research, 55(2), 186-202.

Snowling, M. J., Muter, V., \& Carroll, J. (2007). Children at family risk of dyslexia: A follow-up in early adolescence. Journal of Child Psychology and Psychiatry, 48(6), 609-618.

Soriano-Ferrer, M. (2005). La investigación en dificultades de aprendizaje: Un análisis documental. Neurología, 41, 9, 550-555.

Soriano-Ferrer, M., \& Miranda, A. (2010). Developmental Dyslexia in a Transparent Orthography: A Study of Spanish Dyslexic Children. In Th. E. Scruggs, \& M. A. Mastropieri (Eds.), Literacy and Learning. Advances in Learning and Behavioral Disabilities. Volume 23 (pp. 95-114). Amsterdam: Emerald Group Publishing Limited.
Soriano-Ferrer, M., \& Piedra Martínez, E. (2014). Una revisión de las bases neurobiológicas de la dislexia en población adulta. Neurología, 2014.: http:// dx.doi.org/10.1016/j.nrl.2014.08.003

Swanson L., \& Hsieh C. J. (2009). Reading disabilities in adults: A selective meta-analysis of the literature. Review of Educational Research, 79, 1362-1390.

Swanson, L., (2012). Adults with Reading Disabilities: Converting a Meta-Analysis to Practice. Journal of Learning disabilities, 45(1), 17-30

Tops, W., Callens, M., Lammertyn, T., Hees, V., \& Brisbaert, M. (2012). Identifying students with dyslexia in higher education. Annals of Dyslexia, 62, 186-203.

Undheim, A. M. (2009). A thirteen-year follow-up study of Noewegian adults with dislexia in childhood. Reading Development and educational levels. Dyslexia, 15(4), 291-303.

Wolff, U., \& Lundberg, I. (2003). A Technique for Group Screening of Dyslexia among Adults. Annals of Dyslexia, 53, 324-339. 
